\title{
Differential evolution algorithm for multi-commodity and multi-level of service hub covering location problem
}

\author{
M. EghbaliZarch ${ }^{*}$ M. Abedzadeh and M. Setak
}

Department of Industrial Engineering, University of K.N.Toosi, Tehran, Iran

\begin{tabular}{|c|c|}
\hline ART ICLE I NFO & AB S T RAC T \\
\hline $\begin{array}{l}\text { Article history: } \\
\text { Received 10 August } 2012 \\
\text { Received in revised format } \\
\text { 14 September } 2012 \\
\text { Accepted September } 272012 \\
\text { Available online } \\
\text { 28 September } 2012 \\
\text { Keywords: } \\
\text { Hub covering location } \\
\text { Multi-commodity } \\
\text { Multi-level of service } \\
\text { Differential evolution algorithm } \\
\text { Response surface methodology } \\
\text { Lower and upper bounds }\end{array}$ & $\begin{array}{l}\text { The hub location problem involves a network of origins and destinations over which } \\
\text { transportation takes place. There are many studies associated with finding the location of hub } \\
\text { nodes and the allocation of demand nodes to these located hub nodes to transfer the only one kind } \\
\text { of commodity under one level of service. However, in this study, carrying different commodity } \\
\text { types from origin to destination under various levels of services (e.g. price, punctuality, reliability } \\
\text { or transit time) is studied. Quality of services experienced by users such as speed, convenience, } \\
\text { comfort and security of transportation facilities and services is considered as the level of service. } \\
\text { In each system, different kinds of commodities with various levels of services can be transmitted. } \\
\text { The appropriate level of service that a commodity can be transmitted through is chosen by } \\
\text { customer preferences and the specification of the commodity. So, a mixed integer programming } \\
\text { formulation for single allocation hub covering location problem, which is based on the idea of } \\
\text { transferring multi commodity flows under multi levels of service is presented. These two are } \\
\text { applied concepts, multi-commodity and multi-level of service, which make the model's } \\
\text { assumptions closer to the real world problems. In addition, a differential evolution algorithm is } \\
\text { designed to find near-optimal solutions. The obtained solutions using differential evolution (DE) } \\
\text { algorithm (upper bound), where its parameters are tuned by response surface methodology, are } \\
\text { compared with exact solutions and computed lower bounds by linear relaxation technique to } \\
\text { prove the efficiency of proposed DE algorithm. }\end{array}$ \\
\hline
\end{tabular}

\section{Introduction}

Hub location is extensively applied in transportation (Campbell, 2007), telecommunication (Klincewicz, 1998), postal (Çetiner, 2006) and cargo delivery systems (Alumur \& Kara, 2008a; \& Tan \& Kara, 2007). In these systems, to take advantage of the economies of scale, hubs are intermediates for transmitting the flows instead of sending flows directly among all origin-destination pairs. Hubs are special facilities, which consolidate and distribute the flows.

Hub Location Problem (HLP) is introduced by O'Kelly (1987), which can be classified into single and multiple allocation p-hub median, fixed cost, p-hub center and hub covering (Alumur \& Kara, 2008a). In hub location problem, the primary objective is to find the best location of hubs and the allocation of

* Corresponding author.

E-mail: meghbali@mail.kntu.ac.ir (M. EghbaliZarch)

(C) 2012 Growing Science Ltd. All rights reserved.

doi: 10.5267/j.ijiec.2012.010.001 
demand nodes to located hubs such that optimize the whole network. The primary aim of the p-hub median problem is to minimize the total transportation flow cost between origin-destination pairs and the number of located hubs. In HLP with fixed expenditure, the number of hubs is considered as a decision variable. The p-hub center is to maximize the minimum cost for (1) every origin-destination pair, (2) movement on every origin to hub, hub to hub and hub to destination link and (3) movement between a hub and an origin/destination. In most cases, in hub location problem, every hub pair is connected and there is no direct connection between two demand nodes.

In fact, not all demand nodes can be allocated to each hub. For instance, in air transportation systems low capacity feeder aircraft transport passengers from the low-demand, smaller sized airports to the larger hub airports where passengers are transported to their destinations on bigger capacity aircrafts (Sim, 2007). Hence, capacity feeder limits the number of hub airports where non-hub airports can be assigned to. In postal and cargo delivery systems, customer satisfaction occurs when considered commodities are sent from origin to destination in a promised delivery times (Tan \& Kara, 2007). Therefore, each demand node cannot be allocated to hubs. Campbell (1994) presented hub covering location problem for the first time. Kara and Tansel (2003) suggested linear and Wagner (2004) proposed better formulations. Ernst et al. (2005) introduced cover radius for the first time. Hamacher and Meyer (2006) compared different formulations of HCoP and analyzed its feasibility poly-hedron. They also proposed a binary search, which was based on the inverse relationship between p-hub center and hub covering problem.

Tan and Kara (2007) applied the latest arrival hub covering to the cargo delivery sector in Turkey. Alumur and Kara (2008b) considered hub covering problem over incomplete network allowing at most four hub stops on a route from any origin to destination, while focusing on cargo application. Calik et al. (2009) studied single allocation hub covering problem over incomplete hub networks and proposed a tabu-based heuristic algorithm. Mohammadi et al. (2010) studied single allocation hub covering location problem under a capacity constraint and proposed a hybrid algorithm based on a genetic algorithm and simulated annealing. Mohammadi et al. (2011b) presented a model for capacitated single allocation hub covering location that instead of using capacity constraints to limit the received flow of each hub, the second objective function was introduced to minimize the service times in the hubs and a weighted-based multi-objective imperialist competitive algorithm was designed to find near-optimal solutions.

Mohammadi et al. (2011a) studied the location model for hub covering problem behaving as M/M/C queues and presented a nonlinear formulation and proposed an improved imperialist competitive algorithm to solve the model. Karimi and Bashiri (2011) studied hub covering problem with different coverage types over complete hub networks and proposed two heuristic procedures. Zarandi et al. (2012) studied the multiple allocation hub set-covering problem considering the backup coverage and mandatory dispersion of hubs. Ghodratnama et al. (2012) presented a new fuzzy bi-objective model for hub covering problem with specific features such as considering production facilities, time horizons and transportation vehicles.

Most of the studies in the literature and all of the hub covering location studies in the literature review are dealt with finding the location of hub nodes and the allocation of demand nodes to these located hub nodes to transfer only one kind of commodity under one level of service. In reality, each system that hub location can be applied to consist of multi commodities, which transfer commodities under different levels of services. Quality of services experienced by users such as speed, convenience, comfort and security of transportation facilities and services is considered as levels of service. For instance, in cargo delivery systems, type of commodity can be classified in terms of weight, dimension and nature (like Perishable and non-perishable) that can be transmitted via various kinds of vehicles. In postal systems, letters and categorized parcels according to their weight can be posted by various postal services. 
The Postal Service divides mail into different services, called classes. Each class of mail has different features, postage prices, and presort requirements. For most of mailing systems, the content of the material and postage will determine the selected class of mailing. In air transportation systems, passengers can choose the service level of their preferred aviation company according to ticket prices, non-delay flights, type of airplanes and reliability of services. In telecommunication networks massage or information, in form of voice, data and/or video is transmitted over communication links (e.g. copper cables, radio links or satellite links) (Klincewicz 1998).

In public transportation, each passenger can be traveled under various modes (e.g. walking, driving, bicycling, Commuter Rail / Subway / Bus, taxi, boat). Therefore, in each system with regard to its characteristics, different kinds of commodities with various levels of services can be transmitted. The appropriate level of service that a commodity can be transmitted through is chosen by customer preferences and the specification of the commodities. The innovation of this research was to consider two concepts of multi-commodity \& multi-level of service in hub covering location problem, simultaneously.

This paper presents a hub covering location problem, which is based on the idea of transferring multi commodity flows under multi levels of service. To handle real-size problems, we propose a differential evolution algorithm. This paper is organized as follows. Section 2 elucidates the mathematical model. Section 3 explains the proposed differential evolution algorithm. Section 4 illustrates the computational results on Australian Post (AP) data set. At the end, conclusions are remarked.

\section{Mathematical Model}

The model in this paper is an extension to Ernst and Krishnamoorthy (1999). In the model of Ernst \& Krishnamoorthy (1999) only one kind of commodity under one level of service is transmitted in the network, which is not associated with many real-world problems. Therefore, we apply two indexes ( $n$ $\& m$ ) to distinct different commodities transmitted under various levels of services with different transportation expenditures.

Let $\mathrm{V}$ be a given node set with $v$ nodes. The proposed model locates the pre-determined number of hubs and then assigns non-hub nodes (demand nodes) to locate hubs based on single allocation concept such that each of the origin-hub and hub-destination links meet specified value (cover radius). This model helps to determine the routes for trafficking multi commodity flows under various levels of services from origin to destination through located hubs. The objective of proposed model is to minimize the total cost of flows between any origin-destination points and the total cost of establishing hubs. The applied parameters and variables of the models are summarized in Table 1 and Table 2, respectively.

\section{Table 1}

Model parameters

\begin{tabular}{ll}
\hline Parameter & Description \\
\hline$v$ & Number of nodes \\
$p$ & Number of hubs \\
$\theta$ & Cover radius of each hub \\
$\alpha$ & Discount factor \\
$D_{i k}$ & Distance between node i and node $\mathrm{k}$ \\
$F_{k}$ & Fixed cost of establishing a hub at node $\mathrm{k}$ \\
$C_{i k n m}$ & The unit transportation cost for commodity n under service level m between node i and node $\mathrm{j}$ \\
$W_{i j n m}$ & Demand of commodity n under service level m from node i to node $\mathrm{j}$ \\
$O_{\text {inm }}=\sum_{j} W_{i j n m}$ & Total flow of commodity n under service level m originating at node $\mathrm{i}$ \\
$H_{\text {inm }}=\sum_{j} W_{j i n m}$ & \\
\hline
\end{tabular}


Table 2

Model Variables

\begin{tabular}{|c|c|}
\hline Variable & Description \\
\hline$X_{i k}$ & equals 1 if node $\mathrm{i}$ is allocated to hub $\mathrm{k}$ \\
\hline & Consistent with the literature, if $X_{k \mathrm{k}}=1$, it means that node $\mathrm{k}$ is a hub \\
\hline$Z_{k l}^{i n m}$ & $\begin{array}{l}\text { The total amount of flow of commodity } n \text { under service level } m \text { emanating from node } i \text { that is } \\
\text { routed between hubs } k \text { and } l \text {. }\end{array}$ \\
\hline
\end{tabular}

By considering previous defined parameters and decision variables, the model is formulated as below:

$\operatorname{Min} \sum_{k} F_{k} X_{k k}+\sum_{i} \sum_{k} \sum_{n} \sum_{m} C_{i k n m} X_{i k}\left(O_{i n m}+D_{i n m}\right)+\sum_{i} \sum_{k} \sum_{l} \sum_{n} \sum_{m} \alpha C_{k l n m} Z_{k l}^{i n m}$

subject to:

$\sum_{k} X_{i k}=1 \quad \forall i$

$\sum_{k} X_{k k}=p$

$X_{i k} \leq X_{k k}$

$\forall i, k$

$D_{i k} X_{i k} \leq \theta$

$\forall \mathrm{i}, \mathrm{k}$

$\sum_{j} W_{i j n m} X_{j k}+\sum_{l} Z_{k l}^{i n m}=\sum_{l} Z_{l k}^{i n m}+O_{i n m} X_{i k} \quad \forall i, k, n, m$

$X_{i k} \in\{0,1\} \quad \forall i, k$

$Z_{k l}^{i n m} \geq 0 \quad \forall i, n, m, k, l$

The objective function minimizes total cost of transferring multi commodity flows under various levels of service between any origin-destination points and the total cost of establishing hubs. Eq. (2) ensures that every node is assigned to exactly one hub. Eq. (3) states just $p$ hub can be established. Eq. (4) ensures that node $i$ cannot be assigned to node $k$ unless there is a hub at node $k$. Eq. (5) makes sure that node $i$ can only be allocated to hub $k$, if distance $D_{i k}$ between $i$ and $k$ is at most the radius $\theta$. Eq. (6) is the multi commodity flow balanced under various levels of service equation. Eq. (7) and (8) state that variable $Z_{k l}^{i n m}$ is bigger than zero and $X_{i k}$ is a binary variable.

\section{Differential Evolution algorithm}

In this section the details of the DE algorithm is explained and for better understanding of its procedure, an example is implemented. It is hard to optimally solve most of the NP-complete problems for real-world instances. In this section, a differential evolution algorithm is developed for the presented model. The differential evolution (DE) was introduced by Storn and Price(1996). Nowadays the DE has become one of the most frequently used evolutionary algorithms solving the global optimization problems (e.g. Rao \& Patel, 2012, 2013; Lobato et al., 2012; Vijay Chakaravarthy et al., 2011). Main stages of the DE algorithm are shown in Fig. 1. In the literature of DE algorithm, a parent vector from the current generation is called target vector, a mutant vector obtained through the 
differential mutation operation is known as donor vector and finally an offspring formed by recombining the donor with the target vector is called trial vector (Das \& Suganthan, 2010).

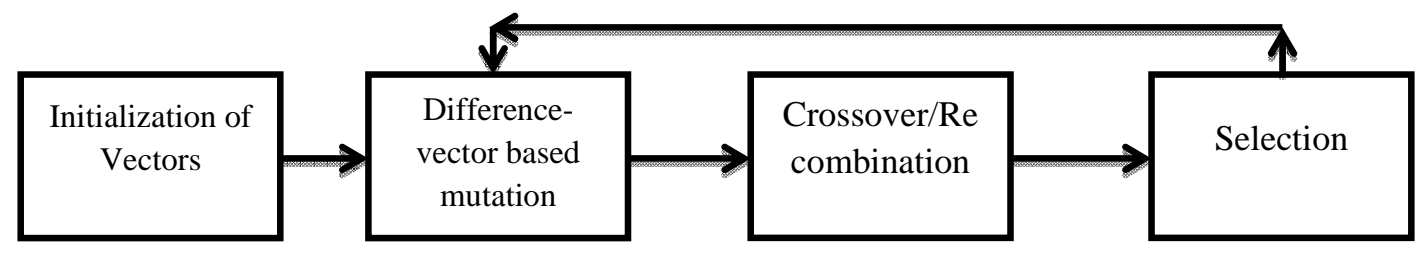

Fig. 1. Main stages of the DE algorithm (Das \&Suganthan, 2010)

\subsection{Representation of the solution}

Any solution encoding procedure should show the location of hub nodes and the allocation of demand nodes to located hub nodes. In this procedure for presenting the given network, some integer numbers are used. The solutions are presented as a matrix. Each column shows a node in the network, in which its elements value explain the number of the hub and the nodes, which are allocated to them. Furthermore, when the value of each element on the entire column is equal to zero, the node is considered as a demand node. If the value of the element that is on the same row and the same column is equal to one, that node is considered as a hub and the rest of the elements show the demand nodes allocated to it. For example, a sample solution is obtained as follows:

$$
S=\left(\begin{array}{llllllll}
0 & 0 & 0 & 0 & 0 & 0 & 1 & 0 \\
0 & 0 & 0 & 0 & 0 & 0 & 1 & 0 \\
0 & 0 & 0 & 0 & 0 & 0 & 0 & 1 \\
0 & 0 & 0 & 0 & 0 & 0 & 0 & 1 \\
0 & 0 & 0 & 0 & 0 & 1 & 0 & 0 \\
0 & 0 & 0 & 0 & 0 & 1 & 0 & 0 \\
0 & 0 & 0 & 0 & 0 & 0 & 1 & 0 \\
0 & 0 & 0 & 0 & 0 & 0 & 0 & 1
\end{array}\right)
$$

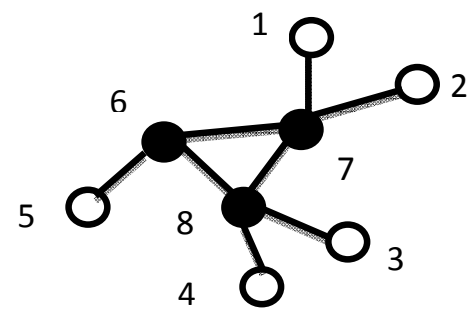

Fig. 2. The representing network of the S solution

In this solution, node 6, 7, 8 are hubs. In addition, node 5 is allocated to hub 6, nodes 1,2 are allocated to hub 7 and nodes 3, 4 are allocated to hub 8.

\subsection{Initial population}

For creating the initial population, first $p$ numbers of hubs are located randomly and form a hub vector. In the allocation step, the remaining nodes are allocated to the located hubs based on their distance from located hubs. If a non-hub node can be allocated to more than one hub, it will be allocated to first hub of mentioned hub vector. After structuring the whole network, the flow balanced constraint will be checked. The above process is applied iteratively to create the entire population.

\subsection{Function evaluation}

The evaluation is an operation to evaluate how good the network structure of each individual is. The evaluation function consists of calculating the objective function value of the network representing by each individual. 


\subsection{Mutation}

Mutation is selected based on the difference of the individual vectors. Randomly selected three individuals $r_{0}, r_{1}$ and $r_{2}$ from a group (in generation g)

$$
V_{i, g}=x_{r_{0}, g}+F \cdot\left(x_{r_{1}, g}-x_{r_{2}, g}\right)
$$

Differential Evolution algorithm is based on real-coded, but this model use 0-1. For binary discrete search space, Kennedy and Eberhart (1997) adapted particle swarm optimization (PSO) to search in binary spaces. PSO applies a sigmoid transformation to the velocity component of Eq. (10) to squash the velocities into a range [0,1], and forces the component values of the locations of particles to be 0 's or 1's.The equation for updating the positions of Eq. (9) is as follows

$$
\begin{aligned}
& \operatorname{sigmoid}\left(V_{i, g}\right)=\frac{1}{1+e^{-V_{i, g}}} \\
& V_{i, g}=\left\{\begin{array}{lr}
1 & \text { if rand }<\operatorname{sigmoid}\left(V_{i, g}\right) \\
0 & \text { otherwise }
\end{array}\right.
\end{aligned}
$$

\subsection{Crossover}

Crossover is to increase the population diversity and ensures that there is a part of the contribution of the previous generation. The crossover operator constructs the offspring $Y_{i, g}$ by mixing components of the current individual $X_{i, g}$ (Target matrix) and the $V_{i, g}$ (mutant matrix) generated by mutation. There are two types of crossover used in DE, binomial and exponential ones. In the proposed algorithm, binomial crossover is applied. Binomial crossover replaces the elements of vector $X_{i, g}$ usingthe following rule:

$$
Y_{i, g}=\left\{\begin{array}{rr}
V_{i, g} & \text { if } \operatorname{rand}_{j}[0,1]<C R \text { or } j=k \\
X_{i, g} & \text { otherwise }
\end{array}\right.
$$

The feasibility of trial matrix that is computed in this stage should be checked. If the trial matrix is not feasible, then it will be necessary to repair it.

\subsection{Repairing procedure}

The solution that a trial matrix is shown should be feasible. The procedure of repairing the solutions is explained as follow:

- If the number of elements with value one on the main diagonal is more than $P$ (more than $p$ hub), then random $P$ elements with value one will be kept and other elements change their values to zero. Otherwise, we randomly change their values from elements on the main diagonal with value zero to one until the elements on the main diagonal with value one will be equal to $P$.

- The zero value of elements on the main diagonal will force the entire column to get zero values.

- If the value of element on the main diagonal is equal to one and sum of its entire row is more than one then the elements, which are not located on the main diagonal will change their values to zero.

- Finally, cover constraint will be checked and if non hub node does not allocated to any hub, then it will be allocated to located hubs according to cover constraint. At the end, flow balanced constraint will be checked.

\subsection{Selection}

To keep the population size constant over subsequent generations, the next step of the algorithm calls for selection to determine whether the target or the trial vector survives to the next generation, i.e., at $\mathrm{G}$ $=\mathrm{G}+1$. The selection operation is based on comparing $Y_{i, g}$ (Trial matrix)and $X_{i, g-1}, X_{i, g}$ is selected. 


$$
X_{i, g}=\left\{\begin{array}{rr}
Y_{i, g} & \text { if } f\left(Y_{i, g}\right) \leq f\left(X_{i . g-1}\right) \\
X_{i, g} & \text { else }
\end{array}\right.
$$

\subsection{Numerical Example}

For more details about this algorithm and its operators, an example with 8 nodes and 3 hubs is explained. From the current population, three individuals are sampled, randomly. Now, a scalar number F scales the differences of any two of these three individuals and the scaled difference is added to the third one to obtain the donor vector $\left(V_{i, g}\right)$. In crossover operator, the donor vector exchanges its components with the target vector. To keep the population size constant over subsequent generations, the next step of the algorithm calls for selection to determine whether the target or the trial vector survives to the next generation (Das \& Suganthan, 2010). This procedure is applied on the mentioned example as follow:

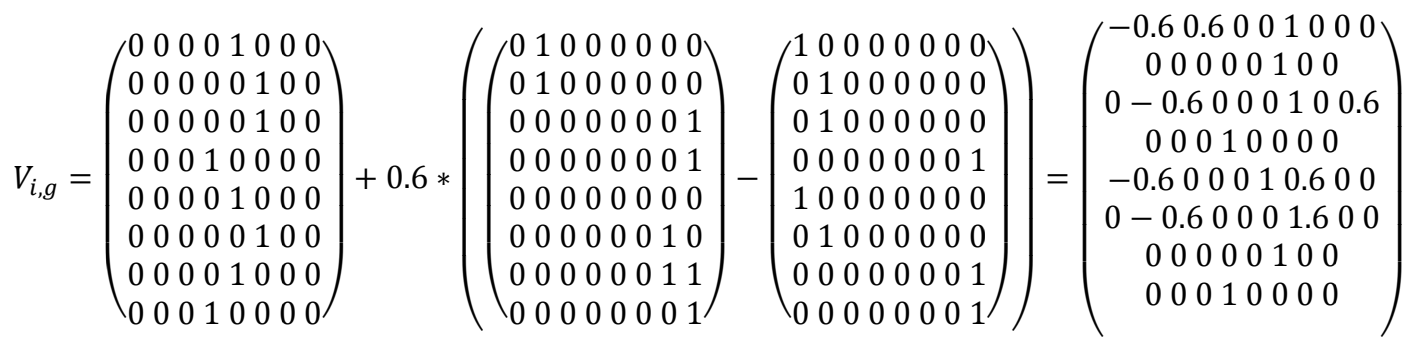

After random sampling, three individuals and added scaled difference of two of these three individuals to the third one are chosen to obtain the donor vector. A sigmoid transformation is applied to the velocity component to squash the velocities into a range $[0,1]$ and forces the component values to be 0 or 1.This procedure shows in Fig. 3.

\begin{tabular}{|c|c|c|c|c|c|c|c|}
\hline 0.6 & 0.6 & 0 & 0 & 1 & 0 & 0 & 0 \\
\hline 0 & 0 & 0 & 0 & 0 & 1 & 0 & 0 \\
\hline 0 & -0.6 & 0 & 0 & 0 & 1 & 0 & 0.6 \\
\hline 0 & 0 & 0 & 1 & 0 & 0 & 0 & 0 \\
\hline 0.0 & 0 & 0 & 0 & 1 & 0.6 & 0 & 0 \\
\hline 0 & -0.6 & 0 & 0 & 0 & 1.6 & 0 & 0 \\
\hline 0 & 0 & 0 & 0 & 1 & 0 & 0 & 0 \\
\hline 0 & 0 & 0 & 1 & 0 & 0 & 0 & 0 \\
\hline
\end{tabular}

\begin{tabular}{|c|c|c|c|c|c|c|c|}
\hline 0.35434 & 0.61566 & 0.5 & 0.5 & 0.73106 & 0.5 & 0.5 & 0.5 \\
\hline 0.5 & 0.5 & 0.5 & 0.5 & 0.5 & 0.73106 & 0.5 & 0.5 \\
\hline 0.5 & 0.5544 & 0.5 & 0.5 & 0.5 & 0.73106 & 0.5 & 0.65566 \\
\hline 0.5 & 0.5 & 0.5 & 0.73106 & 0.5 & 0.5 & 0.5 & 0.5 \\
\hline 0.35434 & 0.5 & 0.5 & 0.5 & 0.73106 & 0.64566 & 0.5 & 0.5 \\
\hline 0.5 & 0.3514 & 0.5 & 0.5 & 0.5 & 0.83202 & 0.5 & 0.5 \\
\hline 0.5 & 0.5 & 0.5 & 0.5 & 0.73106 & 0.5 & 0.5 & 0.5 \\
\hline 0.5 & 0.5 & 0.5 & 0.73106 & 0.5 & 0.5 & 0.5 & 0.5 \\
\hline
\end{tabular}

\begin{tabular}{|c|c|c|c|c|c|c|c|}
\hline 1 & 1 & 1 & 1 & 0 & 1 & 1 & 1 \\
\hline 0 & 1 & 1 & 1 & 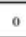 & 1 & 1 & 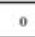 \\
\hline 0 & 1 & 0 & I & 1 & 1 & 0 & $\circ$ \\
\hline ' & 1 & $\circ$ & 1 & $\circ$ & $\circ$ & 0 & 1 \\
\hline 0 & 0 & 0 & 0 & 0 & 1 & 0 & $\circ$ \\
\hline 1 & 1 & $\circ$ & 0 & $\circ$ & 1 & I & 0 \\
\hline 1 & 0 & 0 & 。 & $\circ$ & 1 & 1 & $\circ$ \\
\hline 1 & 1 & 1 & 1 & 1 & 1 & 0 & 8 \\
\hline
\end{tabular}

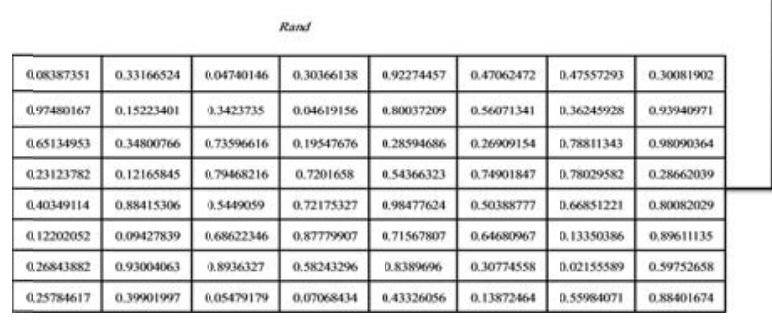

Fig. 3. A sigmoid transformation to the velocity component to squash the velocities into a range $[0,1]$ and force the components values to be 0 or 1 
As shown in Fig. 4, the trial vector is created after mutation and crossover stages. This individual illustrates a solution of the problem and should be entered to the repairing stage for checking its feasibility. First of all, the zero value of elements on the main diagonal will force the entire column to get zero values such as column 1,2,3,7 and 8 . After that, locating $P$ hub is checked (It should be 3 elements on the main diagonal of trial matrix be equal to one). If there is a node not allocated to any hub (the value of the entire specific row is equal to zero), then it will be allocated to one hub according to cover constraint. Checking cover and flow balanced constraint will be performed after all of previous steps.

Target matrix

\begin{tabular}{|c|c|c|c|c|c|c|c|}
\hline 0 & $\circ$ & 0 & 0 & 1 & 0 & 0 & $\circ$ \\
\hline${ }^{\circ}$ & 0 & 0 & $\circ$ & $\circ$ & 1 & ${ }^{\circ}$ & $\overline{0}$ \\
\hline 0 & 0 & 0 & 0 & 0 & ' & 0 & 0 \\
\hline - & 0 & 0 & 1 & 0 & 0 & 0 & 0 \\
\hline o & 0 & 0 & $\circ$ & 1 & 0 & 0 & 0 \\
\hline 0 & 0 & 0 & 0 & 0 & 1 & 0 & 0 \\
\hline 0 & 0 & 0 & 0 & 1 & 0 & 0 & $\circ$ \\
\hline 0 & 0 & 0 & 1 & 0 & 0 & 0 & 0 \\
\hline
\end{tabular}

Mutant matrix

\begin{tabular}{|l|l|l|l|l|l|l|l|}
\hline 1 & 1 & 1 & 1 & 0 & 1 & 1 & 1 \\
\hline 0 & 1 & 1 & 1 & 0 & 1 & 1 & 0 \\
\hline 0 & 1 & 0 & 1 & 1 & 1 & 0 & 0 \\
\hline 1 & 1 & 0 & 1 & 0 & 0 & 0 & 1 \\
\hline 0 & 0 & 0 & 0 & 0 & 1 & 0 & 0 \\
\hline 1 & 1 & 0 & 0 & 0 & 1 & 1 & 0 \\
\hline 1 & 0 & 0 & 0 & 0 & 1 & 1 & 0 \\
\hline 1 & 1 & 1 & 1 & 1 & 1 & 0 & 0 \\
\hline
\end{tabular}

\begin{tabular}{|c|c|c|c|c|c|c|c|}
\hline \multicolumn{1}{|c|}{ Trial matrix } \\
\hline 0 & 1 & 0 & 1 & ${ }^{0}$ & 1 & 1 & 1 \\
\hline 0 & 0 & 1 & 0 & 0 & 1 & 1 & 0 \\
\hline 0 & 0 & 0 & 1 & 0 & 1 & 0 & 0 \\
\hline 0 & 0 & 0 & 0 & 1 & 0 & 0 & 0 \\
\hline 1 & 0 & 0 & 0 & 0 & 1 & 0 & 0 \\
\hline 0 & 0 & 0 & 0 & 0 & 0 & 0 & 0 \\
\hline 1 & 0 & 0 & 1 & 0 & 0 & 0 & 0 \\
\hline
\end{tabular}

\begin{tabular}{|c|c|c|c|c|c|c|c|}
\hline 0.9437315 & 0.3723127 & 0.6684643 & 0.4845483 & 0.2466872 & 0.4503936 & 0.122815 & 0.4979029 \\
\hline 0.5491581 & 0.9371347 & 0.2067765 & 0.7567492 & 0.7844231 & 0.2056723 & 0.4073184 & 0.6948052 \\
\hline 0.7283868 & 0.8295328 & 0.6538506 & 0.4170475 & 0.8828376 & 0.899651 & 0.275287 & 0.834369 \\
\hline 0.5767583 & 0.8490855 & 0.0720516 & 0.971786 & 0.9137117 & 0.7625855 & 0.7166697 & 0.6096297 \\
\hline 0.0258575 & 0.3725342 & 0.4067269 & 0.9879747 & 0.5582849 & 0.8824863 & 0.2833844 & 0.5747372 \\
\hline 0.446531 & 0.5931846 & 0.6669315 & 0.8641475 & 0.5988681 & 0.2849502 & 0.8961989 & 0.3260422 \\
\hline 0.646302 & 0.8725526 & 0.9337257 & 0.3888838 & 0.1488767 & 0.673226 & 0.8265789 & 0.4564246 \\
\hline 0.521203 & 0.9335016 & 0.81095 & 0.4547418 & 0.8997135 & 0.6642799 & 0.3900265 & 0.7137956 \\
\hline
\end{tabular}

Fig. 4. Binomial crossover by mixing components of the current individual $X_{i, g}$ (Target matrix) and the $V_{i, g}$ (mutant matrix)

\section{Computational Result}

In this section, the proposed model is implemented on subsets of AP dataset. AP dataset has been used based on a postal delivery in Sydney, Australia for the first time (Erns \& Krishnamoorthy, 1996). To test the performance of the proposed DE algorithm, a comparison is accomplished between the quality of algorithm solutions (upper bound) and optimal solutions, which is gained by GAMS23.5. We also compare Lower bound computed by linear relaxation (LR) technique on a system with Intel(R) Core ${ }^{\mathrm{TM}}$ Duo T9300 processor $(2.50 \mathrm{GHz})$ with $2 \mathrm{~GB}$ of RAM operating under the system windows.

\subsection{Parameters Tuning}

The quality of an algorithm is significantly affected by the values of its parameters. In this section, response surface methodology (RSM), an approach based on DoE techniques has been used to tune the parameters. This methodology is introduced by Wilson and Box (1951) for the first time. RSM in this 
paper is applied by Design Expert 8 software, which is designed for some experiments to define the promising initial parameter values and leads to improvements in terms of the quality of the solutions.

\subsection{Computing the Cover Constraint}

Eq. (15) explains Sahraeian and Korani's (2010) approach for calculating the cover radius to apply it in the model. By using distance table, the minimum number in each column is obtained (without zero), these values denote vicinity to nodes. Also by choosing maximum number among the minimum numbers of columns, cover radius is found.

$\theta=\max _{j}\left(\min _{i} D_{i j}\right)$

The mentioned dataset does not have data related to commodity and service level type. Hence, Eq. (16) shows how to calculate demand of commodity $n$ under service level $m$ from node $i$ to node $j$ and the unit transportation cost for commodity $n$ under service level $m$ between node $i$ and node $j$ is generated randomly close to unit costs of dataset.

$W_{i j n m}=\frac{W_{i j \text { dataset }}}{n m}$

The results of comparison with different values of parameters are shown in Table 3.

\section{Table 3}

The summary of the performance of the proposed model, GAMS in two circumstances and relative gaps

\begin{tabular}{ccccccc}
\hline \multicolumn{2}{c}{ Parameter } & GAMS(MIP) & DE & GAMS(RMIP) & \multicolumn{2}{c}{ metric } \\
\cline { 1 - 3 } $\mathrm{n}=2, \mathrm{~m}=2$ & $\begin{array}{c}\text { Objective } \\
\text { Function Value }\end{array}$ & Upper bound & Lower bound & Gap1\% & \multirow{2}{*}{ Gap2\% } \\
\hline $\mathrm{v}$ & $\mathrm{p}$ & 633440 & 657863.6816 & 633440 & 3.8 & 3.8 \\
& 5 & 846560 & 846564.0177 & 826710 & 0 & 2.4 \\
& 4 & 1857900 & 1927297.4689 & 1857900 & 3.7 & 3.7 \\
& 5 & 2929400 & 2929400 & 2479300 & 0 & 18.1 \\
25 & 4 & 3774600 & 3896139.0315 & 3460300 & 3.2 & 12.5 \\
& 5 & 5863100 & 5895778.945 & 4169100 & 0.557 & 41.41 \\
40 & 5 & 39291000 & 39450668.4787 & 24279000 & 0.4 & 62.48 \\
& 5 & - & 61331635.3387 & 28761000 & - & 113.2 \\
50 & 5 & - & 68102880.4356 & 38959000 & - & 74.8 \\
& 4 & - & 85400357.3165 & 47759000 & - & 78.8 \\
\hline
\end{tabular}

In this section, five instances with a number of nodes, V, ranging from 15 to 50 are considered. The optimal values of these instances solved by solver GAMS (Cplex) are given in column under optimal objective function value. The column under upper bound give the value of the best solution of DE algorithm and the lower bound column show the value for these instances by relaxing mixed integer programming.

The next two columns under gap1 and gap2 allow us to compare the bounds and give the percent deviation with respect to the optimal solution and lower bound. These two columns consolidate the information of previous columns. These two metrics, which are named gap1 and 2 are computed by: 
gap $1 \%=\frac{O b j_{\text {heiristic }}-O b j_{\text {optimal solution }}}{O b j_{\text {optimal solution }}} \times 100$

gap $2 \%=\frac{O b j_{\text {heiristic }}-\text { lower bound }}{\text { lower bound }} \times 100$

The obtained results show that since it is no longer possible to optimally solve the instances, then the percent deviation between the upper and lower bound is considered. Two mentioned metrics can test the quality of computed bounds and specify how weak they are. Tight bounds resulted from upper and lower bound show how good bounds they are. By considering the small percent deviation of upper bound and optimal solution in small instances and weak lower bound, it can be concluded that the proposed algorithm has acceptable performance.

\section{Conclusion}

In this study, systems carrying different commodity types from origin to destination under various levels of services (e.g. price; punctuality, reliability or transit time) have been studied. For accommodating the hub covering location problem with the real-world data, it is necessary to apply these two concepts (multi-commodity and multi-level of service) in the models. In each system with regard to its characteristics, different kinds of commodity with various levels of service can be transmitted. The appropriate level of service that a commodity can be transmitted through is chosen by customer preferences and the specification of the commodity. In this paper, a mixed integer programming formulation for the hub covering location problem, which is based on the idea of transferring multi commodity flows under multi levels of service is presented. To solve large-sized instances a differential evolution algorithm has been proposed.

The parameters of the algorithm are tuned by response surface methodology. Computational results have compared upper bound (the best solution of DE algorithm) with lower bound and optimal solutions for small instances. The percent deviation between upper and lower bounds shows the quality of computed bounds and specify how weak they are. By considering the small percent deviation of upper bound and optimal solution in small instances and its weak lower bound, it can be concluded that the upper bound has acceptable quality and the proposed algorithm showed a little gap between the solutions obtained by proposed algorithm and GAMS results.

\section{References}

Alumur, SA., \& Kara, B.Y. (2008a). Network hub location: the state of art.European Journal of Operational Research, 1, 1-21.

Alumur, S., \& Kara, B.Y.(2008b). A hub covering network design problem for cargo applications in Turkey. Journal of the Operational Research Society, 10, 1349-1359.

Box, G.E.P., \& Wilson, K.(1951). On the experimental attainment of optimum conditions. Journal of the Royal Statistical Society. Series B (Methodological), 13, 1-45.

Campbell, J.F.(1994). Integer programming formulations of discrete hub location problems.European Journal of Operational Research, 2, 387-405.

Campbell, J.F. (2009). Hub location for time definite transportation. Computers \& Operations Research, 36, 3107-3116.

Calik, H., Alumur, SA., Kara, B.Y. \& Karasan, O.E.,(2009). A tabu-search based heuristic for the hub covering problem over incomplete hub networks. Computers \& Operations Research,12, 30883096. 
Çetiner, S., Sepil, C., \& Süral, H. (2010). Hubbing and routing in postal delivery systems. Annals of Operations Research, 181, 109-124.

Das, S., \& Suganthan, P.N. (2010). Differential evolution: A survey of the state-of-the-art. Evolutionary Computation, IEEE Transactions, 99, 1-28.

Ernst, A., Jiang, H. \& Krishnamoorthy, M.,(2005). Reformulations and computational results for uncapacitated single and multiple allocation hub covering problems.Unpublished Report, CSIRO Mathematical and Information Science.

Ernst, A.T., \& Krishnamoorthy, M. (1996). Efficient algorithms for the uncapacitated single allocation p-hub median problem. Location Science,4, 139-154.

Ernst, A.T., \&Krishnamoorthy, M. (1999). Solution algorithms for the capacitated single allocation hub location problem. Annals of Operations Research, 86, 141-159.

Fazel Zarandi, M., Davari, S.\& Haddad Sisakht, S. (2012). The Q-coverage multiple allocation hub covering problem with mandatory dispersion. Scientia Iranica

Ghodratnama, A., Tavakkoli-Moghaddam, R., \& Azaron, A. (2012). A fuzzy possibilistic bi-objective hub covering problem considering production facilities, time horizons and transporter vehicles. The International Journal of Advanced Manufacturing Technology, 1-20.

Hamacher, H.W., \& Meyer, T. (2006). Hub cover and hub center problems. Departament of Mathematics, University of Kaiserslautern, Germany.

Klincewicz, J.G. (1998). Hub location in backbone/tributary network design: a review. Location Science, 6, 307-335.

Kennedy, J., \&Eberhart, R.C.(1997). A discrete binary version of the particle swarm algorithm. IEEE TRANSACTION.

Karimi, H., \& Bashiri, M. (2011). Hub covering location problems with different coverage types. Scientia Iranica,18, 1571-1578.

Kara, B., \& Tansel, B. (2003). The single-assignment hub covering problem: Models and linearizations.Journal of the Operational Research Society, 59-64.

Lobato, F.S., Gedraite,R. \& Neiro, S.M.S.,(2012). Solution of flow shop scheduling problems using the differential evolution algorithm.3rd International Conference on Engineering Optimization, Rio de Janeiro, Brazil.

Mohammadi, M., Jolai, F. \& Rostami, H. (2011a). An M/M/c queue model for hub covering location problem.Mathematical and Computer Modelling, 54, 2623-2638.

Mohammadi, M., Tavakkoli-Moghaddam, R. \& Rostami, H. (2011b) A multi-objective imperialist competitive algorithm for a capacitated hub covering location problem. International Journal of Industrial Engineering Computations. 2, 671-688.

Mohammadi, M., Tolui, H. \& Yousefi, M. (2010). Solving a hub covering location problem under capacity constraints by a hybrid algorithm. Journal of Applied Operational Research, 2, 109-116.

O'kelly, M.E. (1987). A quadratic integer program for the location of interacting hub facilities.European Journal of Operational Research, 3, 393-404.

Rao, R.V. \& Patel, V.,(2012). An elitist teaching learning based optimization algorithm for solving complex constrained optimization problems.International Journal of Industrial Engineering Computations, 3, 535-560.

Rao, R.V. \& Patel,V. (2013) Comparative performance of an elitist teaching-learning-based optimization algorithm for solving unconstrained optimization problems. International Journal of Industrial Engineering Computations.

Sim, T.K.T. (2007). The hub covering flow problem and the stochastic p-hub center problem. United states: ProQuest Information and Learning Company.

Tan, P.Z., Kara, B.Y. (2007). A hub covering model for cargo delivery systems. Networks, 1, 28-39.

Wagner, B.,(2004) Model formulations for hub covering problems. Journal of the Operational Research Society, 7, 932-938.

Storn, R., \&Price, K., (1996). Minimizing the real functions of the ICEC'96 contest by differential evolution. IEEE Transaction. 
Sahraeian, R., \& Korani, E. (2010). The hierarchical hub maximal covering problem with determinate cover radiuses. IEEE Transaction.

Vijay Chakaravarthy, G., Marimuthu, S., \& Naveen Sait, A. (2011). Performance evaluation of proposed Differential Evolution and Particle Swarm Optimization algorithms for scheduling mmachine flow shops with lot streaming. Journal of Intelligent Manufacturing, 1-17. 